\section{A CASE OF EPIDURAL SPINAL ABSCESS}

\section{J. MINTZMAN, F.R.C.S.}

Further to the article on acute epidural abscess by Leonard Abrahamson, A. A. McConnell, and G. R. Wilson (Journal, June 23rd, p. 1114), I present below the record of a recent case of acute osteomyelitis of spine with epidural abscess. These cases are fairly rare, and very few are published in the British literature.

\section{Case Record}

History.-Mrs. B., aged 38, complained of pain in the middle of the back on November 19th, 1932. The pain increased in severity during the following day. On the first day of the illness she had a temperature of $100^{\circ} \mathrm{F}$., becoming normal on the third day. Pain in the back continued. On the third day of her illness she started vomiting, whici continued for three days. She also complained of very severe bitemporal headache. She was admitted to the National Hospital, Queen Square, under Dr. Gordon Holmes, on November 28th. Her past history was good, but there had been a boil on her chin three weeks before admission.

Examination.-Speech and all cerebral functions were normal, as were all cranial nerves. There was slight but definite pallor of the left optic disk; the right appeared normal. No diplopia, ptosis, strabismus, or exophthalmos. The motor system was normal as regards the upper limbs and trunk, but the patient appeared unable to sit upright without the use of the arms. Motor power was quite good on both sides in the lower limbs. There was disinclination to exercise full motor power in either limb. Reflexes: bicepsjerk, triceps-jerk, and supinator-jerk present right and left. Upper and lower abdominal reflexes absent. Knee- and anklejerks present. Plantar extensor on both sides. No signs of meningeal irritation. Sensation appeared to be normal when tested on admission. Vibration, which was faintly appreciated in both lower limbs, was perhaps slightly more obvious on the right side; it was only faintly appreciated below the tenth dorsal spine.

Progress.-On November 29th the condition of spastic paraplegia, which was present on the previous day, had changed into a marked partial paraplegia of a flaccid type, but with preservation of deep reflexes, which were not accentuated. The right plantar was now extensor and the left flexor. Abdominal reflexes had now disappeared. The intercostal muscles below the ninth on the left side were not contracting, and there was present a relatively sensitive level for pinprick and for vibration at the ninth dorsal nerve segment. Some sensation to pinprick was preserved in the legs. The abdominal muscles did not contract so forcibly below the umbilicus, and the latter was drawn up $2 \mathrm{~cm}$. upon head-raising. This had increased since the previous night. There was tenderness over the lower thoracic and upper lumbar spines. At a later hour of the same day the patient had a complete paraplegia of the flaccid type, with absence of knee- and ankle-jerks. Plantar reflexes were also absent. Sensory examination showed a relatively sensitive level to light touch at the ninth dorsal nerve segment on the left, $2 \mathrm{in}$. above the umbilicus, and in the ninth dorsal segment $1 \mathrm{~cm}$. below the umbilicus on the right. There was now an absolute level for sensation to pinprick at the proximal portion of the second lumbar segment, below which pain was not felt on either side, except down a strip in the antero-lateral portion of the left thigh, in which the pain from the prick was referred to the foot, beneath the left great toe. There was an absolute level at the third lumbar segment for sensation to hot and cold, that for the former being 2 in. higher than that for the latter. Sensation for deep pressure was preserved in the left toe, calf, and thigh, and in the right leg at and above the knee only. In each case it seemed more distinct in the left leg. Sense of position was present for coarse movements at the ankle, but was absent in the great toes. Pathological examination: differential blood cell count showed 19,000 leucocytes on November 29th at 2.30 p.m. Cerebro-spinal fluid: yellow, setting solid jelly-like coagulum; total protein 4 per cent., cells 3 per c.mm. On culture, no growth in twenty-four hours.

Operation.-On November 29th, at 11.45 p.m., Mr. Julian Taylor removed the laminae from the seventh to twelfth dorsal. Yellow creamy pus coated the dura over this region. The pus seemed to trickle round from the ventral and lateral aspects, and more was collected towards the caudal portions of the exposure. The next day, at 10 a.m., there was feeble movement of the toes of the right foot. The kneejerks werc absent, and the left ankle-jerk was present: plantar reflexors? flexor. There was some degree of appreciation of pinprick over both legs. On December 3rd the $=$ was a fair degree of appreciation to pain over the left leg and thigh; there was a slight return of sensation to pinprick in the right leg above the knee. Below the knee the prick was felt only as indefinite touch localized to the right leg. Light touch was not felt in either leg. The patient knew when the left, and perhaps the right, foot was being moved. Otherwise sense of position was entirely absent. Sensation to deep touch was present in both legs, and localized to the proper leg. This was preserved before operation. Reflexes in the legs were absent except for one plantar reflex flicker on the left. A radiogram of the dorsal spine before operation showed no evidence of bone disease. It may be noted that the temperature never rose above $100^{\circ}$, except immediately after operation.

Post-mortem Examination.-The patient died on December 9th. The brain appeared healthy. There was an open laminectomy over the dorsal spine still draining pus. This seemed to be coming from the front of the cord round the left side. On removal of the cord there was evident erosion of the back of the bodies of the first and second lumbar vertebrae. On opening the dura the arachnoid was seen to be infiltrated with purulent lymph over the lumbar enlargement. From pus removed at operation, on November 29th a heavy growth of Staphylococcus aureus was obtained in culture.

\section{Discussion}

This patient gave a definite history of a boil on the chin three weeks before the onset of acute osteomyelitis. The boil was not treated by incision. On first examination paraplegia was found to be present, and an acute transverse lesion of the cord was diagnosed, probably myelitis ; but the rapid change in the patient's condition led to the conclusion that this was a case of compression of spinal cord. The sensory changes were just as predominant as motor changes. There was improvement after operation, but, as the post-mortem demonstrates, she had a ventral abscess resulting from the disease of the bodies of the vertebrae, and had developed spinal meningitis, which caused her death.

I am grateful to Dr. Gordon Holmes and Dr. Greenfield for their kind permission to publish these notes.

G. Faldella, writing in the July 31 st issue of Riv. di Patol. $e$ Clin. Tuberc., maintains that the only truly active and effective method of treating early pulmonary infiltration in its various stages consists in a carefully performed artificial pneumothorax, followed by a good collapse of the lung, continued for a variable time in different cases, but usually only for a short period. Conservative and expectant treatment, if carried on under the supervision of a competent physician under conditions of bodily and mental rest in a modern sanatorium, may be reserved for exceptional cases in which the lesion is in the very earliest stage and there are few or no bacilli in the sputum, no haemoptysis, and no signs of intoxication. Owing to the extreme sensitiveness of these infiltra, tions to stimuli of any kind, it is important to avoia any strain or stimuli of a clinical, climatic, emotional, or other character. Symptomatic remedies may be of value if used carefully. The best treatment for early pulmonary infiltration therefore consists of artificial pneumothorax, a hygienic mode of life, rest, and symptomatic remedies, followed by climatotherapy. 